\title{
O gênero Xylocopa Latreille no Rio Grande do Sul, Brasil (Hymenoptera, Anthophoridae)
}

\author{
Clemens Schlindwein ${ }^{1}$ \\ Boris Schlumpberger ${ }^{2}$ \\ Dieter Wittmann ${ }^{2}$ \\ Jesus Santiago Moure ${ }^{3}$
}

\begin{abstract}
The genus Xylocopa Latreille in Rio Grande do Sul, Brazil (Hymenoptera, Anthophoridae). A survey of the genus Xylocopa Latreille, 1802 is given for Rio Grande do Sul, the southernmost State of Brazil. Data are based on several studies on the bee fauna of southern Brazil and on unpublished observations. A key is provided to the species (males and females) and information on distribution, nesting habits and relation to flowers. Rio Grande do Sul is strikingly rich in species of Xylocopa because of the diversity of habitats and its geographic position in the transition of tropical/subtropical to temperate climate. Nineteen species, classified into ten subgenera, have been recorded in Rio Grande do Sul. Here we maintain the subgenera Ioxylocopa, Megaxylocopa and Xylocospila, which were put into synonymy recently by Minckley (1998). The species are: Xylocopa (Dasyxylocopa) bimaculata Friese, 1903; Xylocopa (Ioxylocopa) chrysopoda Schrottky, 1902; Xylocopa (Megaxylocopa) frontalis (Olivier, 1789); Xylocopa (Nanoxylocopa) ciliata Burmeister, 1876; Xylocopa (Neoxylocopa) augusti Lepeletier, 1841; Xylocopa (N.) brasilianorum (Linnaeus, 1767); Xylocopa (N.) haematospila Moure, 1951; Xylocopa (N.) hirsutissima Maidl, 1912; Xylocopa (N.) nigrocincta Smith, 1854; Xylocopa (N.) ordinaria Smith, 1874; Xylocopa (N.) suspecta Moure \& Camargo, 1988; Xylocopa (N.) tacanensis Moure, 1949; Xylocopa (Schonnherria) macrops Lepeletier, 1841; Xylocopa (S.) simillima Smith, 1854; Xylocopa (S.) splendidula Lepeletier, 1841; Xylocopa (S.) varians Smith, 1874; Xylocopa (Stenoxylocopa) artifex Smith, 1874; Xylocopa (Xylocopoda) elegans Hurd \& Moure, 1963; Xylocopa (Xylocopsis) funesta Maidl, 1912; Xylocopa (Xylocospila) bambusae Schrottky, 1902. Xylocopa tacanensis is for the first time recorded in Brasil.
\end{abstract}

Keywords. Anthophoridae; Hymenoptera; Taxonomy; Xylocopa; Rio Grande do Sul.

\section{INTRODUÇÃO}

O gênero Xylocopa Latreille, 1802 abrange mais que 700 espécies e apresenta distribuição cosmopolita com maior diversidade nas regiões tropicais e subtropicais do novo e velho mundo (Hurd \& MOURE 1963; Hurd 1978A; GERLING et al. 1989). Cerca de 200 espécies são descritas do novo mundo (HuRd \& Moure 1963), 50 do Brasil (HuRd 1978a). Estas abelhas, chamadas de mamangabas (ou mamangavas, mangangás), constroem ninhos em árvores mortas, fazendo galerias ramificadas em troncos e moirões e ninhos não ramificados em ramos delgados ou em caules ocos, como por exemplo em entrenós de bambu ou em inflorescências do "gravatá" (Eryngium Apiaceae).

Em todas as espécies estudadas, as fêmeas-mães são ativas até a cria alcançar a fase adulta (HoGENDOORn 1994). Alimentam com néctar os jovens depois de emergir. Este comportamento representa uma transição entre o nível solitário e social. Em algumas espécies de Xylocopa foi observado o nível subsocial no sentido de Michener (1974). Nestas mamangabas existe uma fêmea reprodutora que convive com outras fêmeas não fecundadas no mesmo ninho (GerLing et al. 1983; Velthuis \& GeRLING 1983). Estas podem ser fêmeas jovens ainda não reprodutivas ou fêmeas velhas que já passaram por uma fase reprodutiva e continuam no mesmo ninho (HoGENDOORN \& Velthuis 1993; 1995).

Dependendo do clima, as mamangabas são uni, bi, ou polivolti-nas (WATMOUGH 1974; 1983). Como outras abelhas, as fêmeas desi-dratam o néctar para aumentar a concentração de açúcar. Wittmann \& Scholz (1989) mostraram que as fêmeas passam o néctar desidratado para seus filhos. Desta maneira, estes conseguem ocupar um território por mais tempo,

\footnotetext{
1. Departamento de Botânica, Universidade Federal de Pernambuco. Av. Prof. Moraes Rêgo s/n, 50670-901 Recife-PE, Brasil. Endereço Eletrônico: schlindw@ufpe.br

2. Institut für Landwirtschaftliche Zoologie und Bienenkunde, Universität Bonn. Melbweg 42, D-53127 Bonn, Alemanha.

3. Departamento de Zoologia, Universidade Federal do Paraná. Caixa Postal 19020, 81531-980 Curitiba-PR, Brasil.
} 
aumentando a probabilidade de atrair uma fêmea virgem.

As informações existentes sobre relações com plantas indicam que as espécies de Xylocopa são poliléticas (LINSLEY et al. 1966; HuRd 1978a, GerLing et al. 1989). No entanto, para muitas espécies suas relações com flores ainda são pouco ou nada conhecidas. As mamangabas são as principais polinizadoras de plantas de diversas famílias (VAN DER PIJL 1954), entre outras de flores de várias espécies de maracujá (Passiflora, Passifloraceae) (SAzima \& Sazima 1989; RoubiK 1995; GARCIA \& Hoc 1997). Fontes importantes de pólen são espécies com anteras poricidas (Solanum, Solanaceae), Cassia, Senna, Chamaecrista (Caesalpiniaceae, Melastomataceae) cujo pólen é coletado por vibração (BuCHMANN 1983). Em flores que apresentam néctar escondido em tubos florais compridos, as mamangabas freqüentemente perfuram o tubo floral com suas gáleas robustas pelo lado de fora e pilham o néctar (SCHREMMER 1972; FAEGRI \& VAN DER PIJL 1979).

Existe, no Brasil, uma carência de chaves para identificação das espécies, regionais ou locais, de abelhas. Trabalhos que fornecem informações sobre a classificação infragenérica e específica são raros, e descrições de espécies novas e estudos sobre a história natural das diversas espécies somente podem ser encontrados em publicações especializadas. Isto dificulta o acesso ao conhecimento da apifauna em geral e o desenvolvimento de estudos, por exemplo da polinização, especialmente quando os pesquisadores não dispõem de uma coleção de referência de abelhas identificadas por especialistas.

Escolhemos, para este trabalho, o gênero Xylocopa, devido à existência de uma revisão do gênero feita por HURD \& MOURE (1963) na qual propuseram novos grupos taxonômicos, com chaves para reconhecê-los. No Brasil, somente havia o trabalho de Sснготтку (1902) para chegar até o nível de espécie.

Baseamo-nos em diversos levantamentos regionais da apifauna no Rio Grande do Sul que forneceram informações sobre a distribuição das espécies, bionomia e comportamento.

Os subgêneros pequenos, Megaxylocopa Olivier, 1789, incluído em Neoxylocopa Michener, 1954 por MinCKLEY (1998) e Ioxylocopa Hurd \& Moure, 1963 e Xylocospila Hurd \& Moure, 1963 incluídos em Schonnherria Lepeletier, 1841 por MINCKLEY (1998) são mantidos aqui.

Para facilitar estudos posteriores apresentamos uma chave para identificação das espécies de mamangabas do Rio Grande do Sul e dados referentes à sua distribuição geográfica e história natural, fornecendo subsídios para futuros estudos de comportamento, distribuição, fisiologia e DNA. A chave e os comentários têm como objetivo principal facilitar o reconhecimento das espécies, sem visar filogenias possíveis como já foi feito no trabalho de HuRd \& MOURE (1963).

\section{MATERIALE MÉTODOS}

Os dados apresentados baseiam-se em estudos regionais da apifauna do Rio Grande do Sul (Scholz 1988; Wittmann \& Scholz 1989; Wittmann \& Hoffmann 1990; Hoffmann 1994; 1995; SChlindwein 1995; 1998; SChlumpberger 1996; Alves dos Santos 1999; Harter 1999; Schlumpberger \& WittManN
2000) e em outras observações não publicadas.

Foram examinados exemplares das coleções: Pontifícia Universidade Católica do Rio Grande do Sul (PUCRS); Fundação Zoobotânica do Rio Grande do Sul, Porto Alegre; Coleção de Entomologia Pe. J. S. Moure, Departamento de Zoologia, Universidade Federal do Paraná, Curitiba (DZUP); Coleção Entomológica do Laboratório de Ecologia Vegetal da Universidade Federal de Pernambuco, Recife.

Os registros das visitas às flores referem-se todos ao Rio Grande do Sul. No Catálogo de HuRD (1978a) há observações complementares sobre flores visitadas.

Abreviaturas utilizadas no texto são: $\mathrm{dp}=$ diâmetro de ponto, $\mathrm{E}=$ esterno, $\mathrm{T}=$ tergo.

\section{RESULTADOS EDISCUSSÃO}

Devido à diversidade de ecossistemas, ocorre um grande número de espécies de Xylocopa no Rio Grande do Sul. O Estado, localizado na transição do clima tropical/subtropical para o temperado, apresenta formações vegetais variadas como a Mata Atlântica, a Mata Pluvial do Alto Uruguai, bem como os campos da Campanha e as florestas semidecíduas da Serra do Sudeste, e o pouco que restou da Mata de Araucária, que se estendia desde o Estado de São Paulo até o Rio Grande do Sul.

Espécies como $X$. (Neoxylocopa) brasilianorum (Linnaeus, 1767) e $X$. (Neoxylocopa) haematospila Moure, 1951 foram somente registradas na Mata Atlântica e na Mata de Araucária; $X$. (Schonnherria) splendidula Lepeletier, 1841 e X. (Neoxylocopa) tacanensis Moure, 1949 ocorrem exclusivamente na Campanha e na Serra do Sudeste. Note-se que este é primeiro registro de $X$. tacanensis no Brasil. Espécies de ampla distribuição na América do Sul como $X$. (Megaxylocopa) frontalis (Olivier, 1789) e X. (Neoxylocopa) nigrocincta Smith, 1854 foram encontradas em todas regiões do Estado.

Há espécies de Xylocopa ligadas a determinado grupo de plantas, não só para alimentação, mas muito mais criticamente à possibilidade de nidificação. Ainda que o substrato mais comum seja troncos mortos, aqui registramos espécies que nidificam em bambu, na mata, e mesmo nas hortas, onde varetas com vários nós de comprimento são usadas como arrimo nas plantações de favas e de outras trepadeiras: X. (Stenoxylocopa) artifex Smith, 1874, X. (Xylocospila) bambusae Schrottky, 1902 e $X$. (Schonnherria) splendidula e também, incidentalmente, $X$. (Schonnherria) macrops Lepeletier, 1841. Muito especializada é X. (Nanoxylcopa) ciliata Burmeister, 1876, que nidifica nas inflorescências mortas do "gravatá", Eryngium (Apiaceae).

Nas espécies de Xylocopa registradas, observaram-se diversas estratégias de acasalamento. Os machos estabelecem territórios, patrulham em áreas longe de recursos florais ou ninhos, ou formam "leks", agregações de vários machos para atrair fêmeas. Patrulhas de machos, em flores preferencialmente visitadas pelas fêmeas, que são comuns em muitas espécies de abelhas solitárias, parecem ser raras em Xylocopa e não foram 
observadas nas espécies ocorrentes no Rio Grande do Sul. Em várias espécies o comportamento reprodutivo ainda não é conhecido. A produção de feromônios para atrair fêmeas é comum. Contudo, na maioria dos casos, não são conhecidas quais as glândulas envolvidas na produção destas substâncias. Em machos de Xylocopa (Dasyxylocopa) bimaculata Friese, 1903 foram encontradas glândulas específicas nos tergos metasomáticos 3 até 5 (SChlumpberger \& WitTMAnN 2000).

\section{ESPÉCIES ESTUDADAS}

Xylocopa (Dasyxylocopa) Hurd \& Moure, 1963

Xylocopa (Dasyxylocopa) Hurd \& Moure, 1963:113 (espécie-tipo: Xylocopa bimaculata Friese, 1903, por designação original).

1.- Xylocopa (Dasyxylocopa) bimaculata Friese, 1903

Xylocopa bimaculata Friese, 1903:202.

Xylocopa (Dasyxylocopa) bimaculata; Hurd \& Moure, 1963:116. Xylocopa piligera Maidl, 1912:327; Hurd, 1978a: 15 (sin.).

Xylocopa leucopus Friese, 1925: 16; Hurd \& Moure, 1963: 116 (sin) Xylocopa (D.) leucopus; Hurd \& Moure, 1963:116.

Distribuição. BRASIL. Goiás. Rio de Janeiro. São Paulo. Paraná. Santa Catarina. Rio Grande do Sul: Caçapava do Sul, Cambará do Sul, Rosário do Sul, São Francisco de Paula e Viamão. PARAGUAI. ARGENTINA: Misiones.

Flores visitadas. Hippeastrum sp. (Amaryllidaceae), Mikania involucrata (Asteraceae), Croton erythroxyloides (Euphorbiaceae), Phaseolus vulgaris (Fabaceae), Tibouchina gracilis (Melastomataceae), Hexachlamys itatiaiensis (Myrtaceae), Fuchsia regia (Onagraceae), Passiflora caerulea (Passifloraceae), Styrax leprosus (Styracaceae).

Nidificação. Não conhecida.

Comportamento. Na primavera os machos pairam em territórios sombreados na borda de mata. Às vezes formam agregações ("leks" ou "leques") de 10 ou mais indivíduos voando separados uns dos outros numa distância de cerca de $50 \mathrm{~cm}$.

Em T3-T5, dorsolateralmente, há glândulas que produzem feromônios. Pairando no ar, contra o vento, os machos repetidamente esfregam as pernas posteriores no metasoma para uma dispersão mais eficiente desses odores. Nesses vôos o território é ocupado até 90 minutos. Depois os machos visitam flores para sugar néctar (SCHLUMPBerger \& WitTMANN 2000).

Notas taxonômicas. Descrita do Rio Grande do Sul. Facilmente reconhecível pelo adorno de pêlos fulvo-amarelados aos lados dos segmentos 5-6, toda pilosidade restante preta. As asas fuscas, muito escuras, com o brilho violeta, não muito bem definido em alguns exemplares.

Xylocopa (Ioxylocopa) Hurd \& Moure, 1963

Xylocopa (Ioxylocopa) Hurd \& Moure, 1963:116 (espécie tipo: Xylocopa chrysopoda Schrottky, 1902, por designação original).
Notas taxonômicas. Subgênero monotípico, em que só o macho é conhecido. Cabeça com grande desenho amarelo na face e a pilosidade também amarela na cabeça, tórax, esternos e fêmures; os tarsos com pilosidade dourada lhe valeram o nome. Schrottky achou possível fosse o macho de X. pulchra, uma Schonnherria. O exemplar-tipo é de Jundiaí $(761 \mathrm{~m})$ e foi coletado pouco atrás da Serra do Mar e está no Museu Paulista. O único exemplar de Curitiba ( $900 \mathrm{~m}$ ), nas matas do planalto curitibano. Em São Francisco de Paula (931 m) só foi observada em área similar, pouco atrás das montanhas do litoral. Apenas a referência a Misiones, Argentina, indica a interiorização da espécie em altitudes mais baixas.

$\mathrm{Na}$ análise cladística feita por MinckLey (1998), este subgênero foi incluído em Schonnherria, que perde assim sua identidade, uma vez que $X$. chrysopoda não tem nenhum brilho metálico, modificando o significado que havia dado a esse grupo de abelhas. Por isso preferimos mantê-lo como unidade sistemática individualizada, no sentido de HuRD \& MOURE (1963).

2.- Xylocopa (Ioxylocopa) chrysopoda Schrottky, 1902 Xylocopa chrysopoda Schrottky, 1902:475.

Xylocopa (Ioxylocopa) chrysopoda; Hurd \& Moure, 1963:118.

Distribuição. BRASIL.São Paulo. Paraná. Santa Catarina. Rio Grande do Sul: São Francisco de Paula. ARGENTINA: Misiones.

Flores visitadas. Não foram observadas relações com flores, mas apenas os vôos dos machos.

Nidificação. Desconhecida.

Comportamento. Abelha rara, pouco coletada. As fêmeas ainda não são conhecidas. Vários machos foram observados ocupando peque-nas áreas ensolaradas de cerca de um metro quadrado, numa altura de 2 até $3 \mathrm{~m}$, nas margens da mata ("non resource based") em São Francisco de Paula. Os machos movimentam-se continuamente nestas áreas sem mostrar agressividade entre si. Todos os locais assinalados não estão afastados da região litorânea.

Xylocopa (Megaxylocopa) Hurd \& Moure, 1963

Xylocopa (Megaxylocopa) Hurd \& Moure, 1963:151, (espécie-tipo: Apis frontalis Olivier, 1789, por designação original).

Notas taxonômicas. Subgênero com três espécies. Duas ocorrem no Brasil: X. frontalis (Olivier, 1789) e X. fimbriata Fabricius, 1804. Esta última, no extremo norte da Amazônia, entra na Colômbia e Venezuela, e chega até o México. $X$. (Megaxylocopa) nautlana Cockerell, 1904, restringe-se a América Central e México.

$\mathrm{Na}$ análise cladística de MincKLey (1998) este grupo foi incluido em Neoxylocopa Michener, 1954, não levando em consideração as modificações essenciais na estrutura da cabeça das fêmeas destas enormes abelhas. MicheNer (2000), na chave, aduz os carateres da fêmea, denomina-a "Megaxylocopa group", afastado de Neoxylocopa, porém segue a orientação de MinCKLEY (loc. cit.), suprimindo-o como subgênero [Moure]. 
3.- Xylocopa (Megaxylocopa) frontalis (Olivier, 1789)

Apis frontalis Olivier, 1789:64

Xylocopa nitens Lepeletier, 1841:176; Hurd, 1978a: 81 (sin.)

Xylocopa fasciata Lepeletier, 1841: 202; Smith, 1874: 284 (sin.);

Taschenberg, 1879: 569; Schrottky, 1902: 462.

Xylocopa quadrimaculata Meunier, 1892:64; Hurd, 1978a: 81 (sin.).

Xylocopa morio callichlora Cockerell, 1911:287; Hurd, 1978a: 81(sin.).

Xylocopa frontalis coeruleomicans Enderlein, 1913: 158; Hurd, 1978a: 81 ( $\sin$.)

Xylocopa frontalis viridimicans Enderlein, 1913: 158; Hurd, 1978a: 81 (sin).

Xylocopa frontalis fabricii Cockerell, 1926: 658; Hurd, 1978a: 81 (sin.). Xylocopa frontalis roseata Cockerell, 1926: 658; Hurd, 1978a: 81 (sin.). Xylocopa frontalis trinitatis Cockerell, 1926: 658; Hurd, 1978a: 81 (sin.).

Xylocopa frontalis purpureipennis Cockerell, 1949: 484; Hurd, 1978a: $81(\sin$.$) .$

Xylocopa frontalis obscuripennis Cockerell, 1949: 484; Hurd, 1978a: $82(\sin )$.

Xylocopa americana Prance, 1977(1976):238. Nom. nudum, Hurd, 1978a: 82 (sin).

Distribuição. BRASIL. Rio Grande do Sul: Caçapava do Sul, Guaíba, Nova Petrópolis, Pelotas, Porto Alegre, Quaraí, Rio Grande, Santana da Boa Vista, São Lourenço do Sul, São Marcos e Viamão.

Há citações como, da Argentina ao México, incluindo todo Brasil, entrando nos Andes ocidentais desde o Peru à Colômbia, e no Nordeste em Trinidad, Guiana e Venezuela. A distribuição ao Norte vai do Panamá até o México Central.

Flores visitadas. Helianthus annuus (Asteraceae), Tabebuia chrysotricha, T. umbellata (Bignoniaceae), Senna macranthera (Caesalpiniaceae), Canavalia paraguariensis, Phaseolus vulgaris (Fabaceae), Sinningia macrostachya (Gesneriaceae), Leonurus sibiricus (Lamiaceae), Tibouchina gracilis (Melastomataceae), Melia azedarach (Meliaceae), Passiflora caerulea (Passifloraceae), Serjania meridionalis (Sapindaceae), Styrax leprosus (Styracaceae), Aloysia gratissima, Stachytarpheta cayennensis (Verbenaceae).

\section{Nidificação. Madeira morta.}

Comportamento. Os machos ocupam territórios com 1-2 m, voando em círculos ("non resource sites"). É uma das maiores abelhas da região neotropical (algumas fêmeas medem até 36 $\mathrm{mm}$ ), comum e com ampla distribuição. É freqüente sua ocorrência em áreas urbanas. Dimorfismo sexual acentuado: as fêmeas pretas e os machos fulvo-amarelados. Durante o período de acasalamento, os machos jovens retornam inicialmente ao ninho maternal onde são alimentados pela mãe. Depois de alguns dias passam a ocupar ninhos abandonados e vivem isolados das fêmeas nas horas de vôo, e coletam néctar em flores.

Notas taxonômicas. Deu-se grande importância ao colorido das asas; nos exemplares do Brasil as asas são escuras com reflexos violáceos fortes. Para variações no colorido e reflexos das asas foram dados alguns nomes com registro no Catálogo de HuRD, 1978a. Realmente as asas de colorido pálido com fortes reflexos esverdeados, como ocorre na Colômbia dão um visual muito diferente a espécie, e parece justificar callichlora. Veja-se a consideração de Pérez (1901), para a cor das asas, que permitiriam aproveitar alguns desses nomes para designar raças geográficas e mesmo confirmar-se como de valor subespecífico ou específico por levantamentos mais detalhados nas regiões em que ocorrem. ENDERLEIN (1913) registra a espécie como $X$. coeruleomicans, com reflexos azuis no Estado do Espírito Santo.

A separação dos machos é fácil devido ao seu grande tamanho e as faixas tergais pretas largas, que em muitos exemplares cobrem o tergo por completo.

Xylocopa (Nanoxylocopa) Hurd \& Moure, 1963

Xylocopa (Nanoxylocopa) Hurd \& Moure, 1963:99 (espécie-tipo: Xylocopa ciliata Burmeister, 1876, por designação original).

Notas taxonômicas. Subgênero monotípico, apresenta afinidades com Schonnherria. Abelhas pretas, não metálicas, de porte pequeno (comprimento $16 \mathrm{~mm}$ ). Os machos diferem pelas tégulas uniformemente pontuadas e desenhos brancos na metade inferior da face; as fêmeas pela presença de um pequeno tubérculo transversal central na fronte, isolado, sem carena de ligação ao ocelo médio.

4.- Xylocopa (Nanoxylocopa) ciliata Burmeister, 1876 Xylocopa ciliata Burmeister, 1876:158.

Xylocopa (Nanoxylocopa) ciliata Hurd \& Moure, 1963:102.

Xylocopa cavicornis Pérez, 1901: 70; Brèthes, 1916: 410 (sin.); Hurd \& Moure, 1961: 183.

Distribuição. BRASIL. Rio Grande do Sul: Guaiba, Caçapava do Sul, Pinheiro Machado, Porto Alegre, Quaraí, Santana do Livramento e São Francisco de Paula. BOLÍVIA. PARAGUAI. URUGUAI. ARGENTINA.

Conhece-se dos três Estados sulinos nos campos abertos onde ocorrem diversas espécies do gênero Eryngium (Apiaceae, "caraguatá" "gravatá") em cujas inflorescências nidifica. Certamente sua distribuição está vinculada à dispersão destas espécies de planta. A sua sobrevivência é fortemente influenciada pela pela prática de queimadas anuais das pastagens, que destroi seus locais de nidificação.

Flores visitadas. Aspilia montevidensis, Calea phyllolepis, Hypochoeris radicata (Asteraceae), Buddleja thyrsoides (Buddlejaceae) Macroptilium prostratum (Fabaceae), Peltodon longipes (Lamiaceae), Monnina cuneata (Polygalaceae).

Nidificação. Inflorescências mortas de Eryngium (Apiaceae).

Comportamento. Não foram anotadas particularidades no seu comportamento.

Xylocopa (Neoxylocopa) Michener, 1954

Neoxylocopa Michener, 1954:157 (espécie-tipo: Apis brasilianorum Linnaeus, 1787, por designação original).

Xylocopa (Neoxylocopa); Hurd \& Moure, 1963:146; Hurd, 1978a:45.

É o maior subgênero da Região Neotropical com pelo menos 50 espécies descritas, o mais diversificado e com maior distribuição geográfica com $X$. darwini chegando a Galápagos, $X$. sonorina ao Hawai e $X$. varipuncta aos Estados Unidos.

No Rio Grande do Sul ocorrem sete espécies. 
O dimorfismo sexual é muito acentuado: as fêmeas têm o tegumento preto; a pilosidade uniformemente preta é substituida parcialmente em algumas espécies por pilosidade branca ou vermelho-ferrugínea no tórax, ou por franjas abdominais vermelhas. Os machos têm o tegumento fulvoamarelento com alguns desenhos pretos. Nos machos a configuração da genitália permite separar as espécies, porém as espécies são difíceis de reconhecer e deve-se prestar atenção em ninhos e acasalamento, para assegurar a união taxonômica dos sexos.

5.- Xylocopa (Neoxylocopa) augusti Lepeletier, 1841.

Xylocopa augusti Lepeletier, 1841: 187.

Xylocopa (Neoxylocopa) augusti; Hurd \& Moure, 1963: 151.

Xylocopa ferruginea Lepeletier, 1841:187; Hurd \& Moure 1961: 189 (praeocc.)

Xylocopa augusti var. pterochloris Brèthes, 1916: 414

Xylocopa guaranitica Brèthes, 1916: 415.

Distribuição. BRASIL. Goiás. São Paulo. Paraná. Santa Catarina. Rio Grande do Sul: Caçapava do Sul, Canela, Canguçu, Gramado, Guaiba, Lavras do Sul, Nova Petrópolis, Pinheiro Machado, Piratini, Porto Alegre, Quaraí, Rio Grande, Santana da Boa Vista, Santana do Livramento, São Francisco de Paula, Uruguaiana e Viamão. PARAGUAI. ARGENTINA.

Flores visitadas. Oxypetalum sp. (Asclepiadaceae), Carelia cistifolia, Eupatorium intermedium, Hypochoeris pampasica, Vernonia nudiflora (Asteraceae), Macfadyena unguiscati, Pithecoctenium echinatum, Tabebuia umbellata (Bignoniaceae), Opuntia bonaerensis, O. brunneogemmia (Cactaceae), Cassia grandis, Senna macranthera (Caesalpinaceae), Lupinus gibertianus, Phaseolus vulgaris (Fabaceae), Salvia brevipes (Lamiaceae), Nectandra lancolata (Lauraceae), Ligaria cuneifolia (Loranthaceae), Melia azedarach (Meliaceae), Campomanesia aurea, Eugenia uniflora (Myrtaceae), Passiflora caerulea, P. foetida (Passifloraceae), Borreria verbenoides (Rubiaceae), Petunia axillaris, Solanum atripurpureum, S. sisymbriifolium (Solanaceae), Styrax leprosus (Styracaceae), Symplocus uniflora (Symplocaceae), Aloysia gratissima (Verbenaceae).

Nidificação. Em madeira morta (também ramos secos de árvores vivas), árvores caídas, troncos, madeira de construção, postes, latadas de parreirais, etc.

Não foram feitas notas sobre seu comportamento. URBAN (1963) estudou a musculatura cefálica, completada por trabalhos de GRAF (1965, 1968 e 1972). As fêmeas são facilmente reconhecíveis pelas largas franjas de pêlos vermelhos que revestem os lados do abdóme.

Os exemplares-tipos de $X$. guaranitica foram vistos em Buenos Aires por Moure.

6.- Xylocopa (Neoxylocopa) brasilianorum (Linnaeus, 1767) Apis brasilianorum Linnaeus, 1767:961.

Apis brasiliana Linnaeus, 1775: 905.

Xylocopa (Neoxylocopa) brasilianorum; Hurd \& Moure, 1963:151.
Canela, Capão da Canoa, Osório e São Francisco de Paula.

Para outras referências veja-se HuRd (1978a). Autores anteriores interpretaram mal a espécie e atribuíram-lhe uma dispersão enorme. Veja-se o comentário de Pérez (1901).

Flores visitadas. Vernonia constricta, V. grandis (Asteraceae), Anemopaegma prostratum (Bignoniaceae), Opuntia monacantha (Cactaceae), Canna indica (Cannaceae), Ipomoea congesta (Convolvulaceae), Erythrina cristagalli, Lupinus setifolius (Fabaceae), Tibouchina gracilis, T. grandis (Melastomataceae), Myrcia obtecta (Myrtaceae), Fuchsia regia, Ludwigia longifolia (Onagraceae), Passiflora caerulea (Passifloraceae), Pontederia lanceolata (Pontederiaceae), Escallonia chlorophylla (Saxifragaceae), Cyphomandra corymbiflora, Solanum atropurpureum, S. flaccidium, S. variabile (Solanaceae).

Nidificação. Em madeira morta.

Comportamento. Os machos formam territórios pequenos nas margens da mata, entre $50 \mathrm{~cm}$ a $6 \mathrm{~m}$ de altura. Muitas vezes vários machos estabelecem áreas próximas umas das outras sem apresentar agressividade entre si. Ocorrem vôos rápidos em grandes faixas ou círculos, alternando com fases nas quais ficam pairando no ar. Espécie exclusiva da Mata Atlântica principalmente onde predomina a araucária.

Notas taxonômicas. A interpretação da espécie tem sido a mais variada. A interpretação que Moure vem mantendo para esta espécie, restringe a sua distribuição à Mata Atlântica, onde chega pelo norte até a Bahia e pelo sul até o Rio Grande do Sul. As fêmeas, inteiramente pretas e preto-pilosas, as asas moderadamente pardo-claras com reflexos cúpreo-purpúreos bastante intensos em certa luz na parte apical livre de células. $\mathrm{O}$ escutelo largamente liso é suavemente curvo atrás; T1 plumoso-piloso, os demais com cerdas simples; T2 com pontuação relativamente densa (no disco para a base os intervalos cerca de dois pontos porém bastante mais curtos

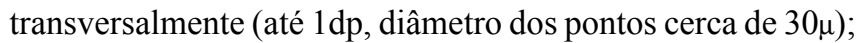
as cerdas curtas, semi-eretas no disco com aproximadamente $300 \mu$; os intervalos densamente micro-pontilhados. Não julgamos fidedignas provenências mais ao norte, principalmente do Caribe. Moure coletou exemplares no Espírito Santo e ao Sul de Salvador, na Bahia. Veja-se os comentários de Hurd (1978a).

\section{7.- Xylocopa (Neoxylocopa) haematospila Moure,1951}

Xylocopa haematospila Moure, 1951:318

Distribuição. BRASIL. Paraná: Marumbi. Santa Catarina: Blumenau. Rio Grande do Sul: São Francisco de Paula.

Flores visitadas. Passiflora caerulea (Passifloraceae), Cyphomandra corymbiflora (Solanaceae). No Paraná o tipo foi coletado em flores de uma Caesalpiniaceae.

Nidificação. Desconhecida. 
Comportamento. Não observado.

Notas taxonômicas. Espécie rara do Sul do Brasil, ocorrendo na Mata de Araucária e Mata Atlântica tendo sido descrita da encosta oriental da Serra do Mar, no Estado do Paraná, na antiga estrada da Graciosa que liga Curitiba a Paranaguá.

Muito parecida com $X$. brasilianorum, separando-se facilmente pela mancha cor de sangue abaixo da placa basitibial, formada por pêlos desta cor. Se excetuamos essa mancha, $X$. haematospila, é em tudo semelhante a $X$. brasilianorum como a interpretou Hurd.

No Museu de Londres, Moure estudou exemplares fêmeas de X. haematospila de Santa Catarina, coletados por Crowley em 1901, um exemplar do Paraná, uma fêmea do Rio Grande do Sul, mandada por Ihering (1884-16) e mais uma fêmea de Asunción, Paraguai, coletada pela Missão Científica Brasil (xi/ 1943). Este último exemplar foi identificado com menos segurança por ter as asas um pouco mais escuras que o normal.

8. - Xylocopa (Neoxylocopa) nigrocincta Smith, 1854 Xylocopa nigro-cincta Smith, 1854:354.

Xylocopa (Neoxylocopa) nigro-cincta; Hurd \& Moure, 1963:151.

Xylocopa schulthesii Dusmet \& Alonso, 1924:52.

Xylocopa (N.) schultessii; Hurd \& Moure, 1963:151.

Distribuição. BRASIL. Bahia. Mato Grosso. Minas Gerais. Espirito Santo. Rio de Janeiro. Santa Catarina. São Paulo. Paraná. Rio Grande do Sul: Caçapava do Sul, Canela, Guaíba, Nova Petrópolis, Porto Alegre, São Marcos, Viamão. PARAGUAI. ARGENTINA, Misiones.

Flores visitadas. Helianthus annuus, Vernonia nudiflora (Asteraceae), Raphanus raphanistrum (Brassicaceae), Bauhinia forficata, Cassia grandis, Senna macranthera (Caesalpiniaceae), Phaseolus vulgaris (Fabaceae), Salvia sp. (Lamiaceae), Tibouchina asperior (Melastomataceae), Melia azedarach (Meliaceae), Jasminum nudiflorum (Oleaceae), Styrax leprosus (Styracaceae), Duranta repens, Stachytarpheta cayennensis (Verbenaceae).

Nidificação. Em madeira morta, ramos secos de árvores vivas, árvores mortas caidas.

Comportamento. Espécie comum em todo o Estado, ocorre também em ambientes urbanos. Os machos estabelecem territórios em copas de árvores fora da época de floração. Durante todo o período de acasalamento, voltam ao ninho maternal onde são alimentados com néctar coletado pela mãe e pelas irmãs. No ninho, encontra-se pelo menos uma fêmea inativa que recebe o néctar da mãe. Esta fêmea desidrata o néctar, passando-o aos machos; estes também desidratam néctar. Foi mostrado que machos alimentados com néctar desidratado ocupam territórios por mais tempo do que aqueles que receberam néctar menos concentrado (WITTMAnN \& SchOLZ, 1989).

Notas taxonômicas. As fêmeas são pretas, com pilosidade preta, porem os tergos abdominais com faixas avermelhadas, as vezes pouco nítidas.
9.- Xylocopa (Neoxylocopa) ordinaria Smith, 1874

Xylocopa ordinaria Smith, 1874:292.

Xylocopa (Neoxylocopa) ordinaria; Hurd \& Moure, 1963:151.

Distribuição. BRASIL. Mato Grossso do Sul. Rio de Janeiro. Espirito Santo. Rio Grande do Sul: São Francisco de Paula. ARGENTINA (?)

Flores visitadas. Desmodium sp. (Fabaceae).

Nidificação. desconhecida.

Notas taxonômicas. Além da cor das asas, o carácter mais óbvio para separar esta espécie de $X$. suspecta está na pontuação dos tergos, principalmente no disco do segundo tergo, onde os pontos esparsos com pêlos curtíssimos de $X$. suspecta, passam a bastante mais longos e fortes e muito mais densos, com pêlos sobrepassando o bordo dos pontos, e a margem lisa desse tergo bem contrastada (Moure \& CAMARGO 1988). Exemplares de Dourados (430 m), Estado de Mato Grosso do Sul, em nada diferem dos do Rio de Janeiro, cerca de 2.400 $\mathrm{km}$ para o Oeste. As notas de Moure sobre o tipo no Museu Britânico[31-Aug. 1958] dizem ser o "escutelo em ângulo obtuso fechado com o bordo de passagem delicadamente arredondado; a pontuação no disco dos T2-3 com cerdas curtas sem atingir o ponto seguinte, em T4-5 mais longas; a pilosidade adensandose para os lados de T2-5. Asas fortemente violáceas. Carena ventral muito forte."

10.-Xylocopa (Neoxylocopa) suspecta Moure \& Camargo, 1988 Xylocopa (Neoxylocopa) suspecta Moure \& Camargo, 1988:209.

Distribuição. BRASIL. Pernambuco. São Paulo, Rio de Janeiro. Rio Grande do Sul: São Francisco de Paula. ARGENTINA. BOLÍVIA. URUGUAI. PARAGUAI.

Flores visitadas. Passiflora caerulea (Passifloraceae).

Nidificação. Madeira morta.

Notas taxonômicas. Esta espécie achava-se identificada nas coleções como $X$. virescens, devido ao forte brilho verde das asas. Moure, ao estudar o tipo de $X$. virescens Lepeletier, 1841, na coleção de Serville, no Instituto de Zoologia da Universidade de Turin definiu-a como "uma $X$. (Megaxylocopa) fimbriata de cornos curtos". No trabalho de Moure \& CAMARgo (1988) foi feita uma descrição detalhada como $X$. suspecta, destacando os carateres referentes ao colorido das asas e estrutura do escutelo e tergos abdominais. As asas bastante escuras com fortes reflexos verde-metálicos, às vezes com pequenas porções próximas ao bordo anterior com reflexos parcialmente violáceos; o escutelo com a parte dorsal em ângulo com a vertical, porem o encontro entre as duas partes sem carena mas curtíssimamente arredondado; a pilosidade de T1 plumosa, em T2 muito curta, apenas sobressaindo ao ponto, algumas cerdas até cerca de duas a três vezes esse diâmetro; os pontos de T2 entre 10 e $20 \mu$, no disco muito esparsa (intervalos até 10/12 diâmetros de ponto), mais adensada para os lados; a superfície suavemente micro-reticulada. [Moure]. 
11.- Xylocopa (Neoxylocopa) tacanensis Moure, 1949

Xylocopa (Neoxylocopa) tacanensis Moure, 1949:457.

Distribuição. BRASIL. Rio Grande do Sul: Quarai. ARGENTINA Os tipos foram descritos de Tacanas, Tucumán, na Argentina.

Flores visitadas. Não foram feitas anotações.

Nidificação. Não conhecida.

Notas taxonômicas. A espécie era conhecida somente da Província de Tucumán, na Argentina. Esta é a primeira vez que se faz menção para o Brasil. No Rio Grande do Sul ocorre exclusivamente na campanha.

Assemelha-se a $X$. aurulenta (FABRicius, 1804:451) pela pilosidade vermelha, porém afasta-se completamente pelo colorido das asas (MOURE 1960:143). É facilmente separável de X. eximia Pérez, 1901 e X. rufidorsum Enderlein, 1913, pela coloração ferrugíneo-clara que reveste o dorso do tórax sem chegar aos lobos nem aos mesepisternos, e pelas tégulas pretas, que nas duas espécies citadas as têm claro-ferrugíneas.

Xylocopa (Schonnherria) Lepeletier, 1841

Schonnherria Lepeletier, 1841:207 (espécie-tipo: Xylocopa micans Lepeletier, 1841, por designação subseqüente); Michener, 1997:54; Minckley, 1998:36; Michener, 2000:590.

Schönherria [sic]; DallaTorre, 1896: 202; Ashmead, 1899:71; Sandhouse, 1943:598

Xylocopa (Schoenherria) [sic]; Hurd \& Moure, 1963:118; Hurd, 1978a: 15.

É o segundo maior subgênero de Xylocopa na Região Neotropical com 29 nomes para espécies e subespécies registrados no catálogo de HuRD (1978a). No Rio Grande do Sul ocorrem 4 espécies e no Brasil são conhecidas pelo menos 17 espécies. São mamangabas geralmente de porte médio com o tegumento realçado pelo brilho metálico. Moure viu os tipos de Maidl no Museu de Viena [1958].

$\mathrm{Na}$ época em que foi publicado o nome como sendo um novo grupo (LEPELETIER 1841: 207) foi impresso "Schonnherria" como citado em Minckley (1998) e Michener (2000). HuRd \& MOURE (1963) verificaram que o nome estava grafado errado (nome do entomólogo alemão Carl Johann Schönherr 17721848 que também assinou trabalhos como Schoenherr). HuRD \& MOURE (1963) justificaram a correção do nome seguindo os artigos 27 e 32c do International Code of Zoological Nomenclature. Como a Comissão Internacional de Nomenclatura Zoológica não foi consultada formalmente prevalece a grafia errada "Schonnherria".

12.- Xylocopa (Schonnherria) macrops Lepeletier, 1841 Xylocopa macrops Lepeletier, 1841:209.

Xylocopa (Schoenherria [sic]) macrops; Hurd \& Moure, 1963:123. Xylocopa crotalariae Schrottky, 1901:212. Hurd \& Moure, 1963: 191 (sin.).

Distribuição. BRASIL. Pará. Amazonas. Ceará. Pernambuco. Mato Grosso. Goiás. Brasília. Bahia. Minas Gerais. Espirito Santo. Rio de Janeiro. São Paulo. Paraná. Santa Catarina. Rio Grande do Sul:
Alegrete, Caçapava do Sul, Osório, Porto Alegre, Rosário do Sul e Viamão. COLÔMBIA. BOLÍVIA. PERU. PARAGUAI. ARGENTINA.

Flores visitadas. Crotalaria sp. (Fabaceae), Allophylus edulis (Sapindaceae).

Nidificação. Foram vistos ninhos em inflorescências de Eryngium. A espécie ocorre na metade sul do estado.

Comportamento. Um macho foi observado procurando fêmeas em inflorescências de uma Lamiaceae. Durante todo o período de acasalamento, os machos foram observados retornando ao ninho maternal onde são alimentados pelas fêmeas. Visitas às flores foram esporádicas.

\section{3.- Xylocopa (Schonnherria) simillima Smith, 1854}

Xylocopa simillima Smith, 1854:357.

Xylocopa (Schoenherria [sic]) simillima; Hurd \& Moure, 1963:123.

Xylocopa mendax Maidl, 1912:319; Hurd \& Moure, 1961: 191 (sin.).

Xylocopa rotundiscuta Brèthes, 1916:418; Hurd \& Moure, 1961: 191 $(\sin )$.

Distribuição. BRASIL. Amazonas. Acre. Pará. Minas Gerais. São Paulo. Paraná. Santa Catarina. Rio Grande do Sul: no litoral próximo a Osório. PERU. BOLÍVIA. PARAGUAI. ARGENTINA.

Nidificação. Não conhecida.

Flores visitadas. Não conhecidas no Rio Grande do Sul.

14.- Xylocopa (Schonnherria) splendidula Lepeletier, 1841 Xylocopa (Schonnherria) splendidula Lepeletier, 1841:190.

Xylocopa (Schoenherria [sic]) splendidula; Hurd \& Moure, 1963:123.

Distribuição. BRASIL. Minas Gerais. Espirito Santo. São Paulo. Paraná. Rio Grande do Sul: Quaraí. Ocorre somente na Campanha. BOLÍVIA. PARAGUAI. ARGENTINA.

Flores visitadas. Não conhecidas

Nidificação. Em madeira morta e em entrenós de bambu.

Nota taxonômica. Tem um forte colorido azul metálico e o porte está ao redor de $15-16 \mathrm{~mm}$.

\section{5.- Xylocopa (Schonnherria) varians Smith, 1874}

Xylocopa varians Smith, 1874:291.

Xylocopa (Schoenherria [sic]) varians; Hurd \& Moure, 1963:123.

Distribuição. BRASIL. Amazonas. Paraíba. Mato Grosso. Espirito Santo. Rio de Janeiro. São Paulo. Paraná. Santa Catarina. Rio Grande do Sul: Porto Alegre, Esteio, Osório e Rio Grande. BOLÍVIA. PARAGUAI. ARGENTINA.

Flores visitadas. Tibouchina sp. (Melastomataceae).

Nidificação. Em madeira morta.

Comportamento. Já na primeira saida do ninho, os machos procuram ninhos abandonados, inclusive de outras espécies. Este ninho é ocupado durante todo período de acasalamento, 
às vezes junto com outros machos.

Xylocopa (Stenoxylocopa) Hurd \& Moure, 1960

Stenoxylocopa Hurd \& Moure, 1960:156 (espécie-tipo: Xylocopa artifex Smith, 1874, por designação original)

Xylocopa (Stenoxylocopa); Hurd \& Moure, 1963:156.

Notas taxonômicas. Subgênero com 5 espécies descritas (HuRd 1978b). X. artifex foi mal interpretada por vários pesquisadores. A espécie é comum do litoral norte do RS e da Mata Atlântica, ocorre também em vegetação aberta e ambiente urbano. Esta espécie está ligada à presença de bambú na mata e nas hortas. No sul ocorre uma espécie menor, X. nogueirai, ainda não constatada no Rio Grande do Sul. No sul da Colômbia o gênero está representado por X. lehmanni Friese, 1903 e chega à America Central com $X$. strandi Dusmet y Alonso, 1924, na Costa Rica.

16.- Xylocopa (Stenoxylocopa) artifex Smith, 1874 Xylocopa artifex Smith, 1874:289.

Xylocopa (Stenoxylocopa) artifex; Hurd \& Moure, 1963:160.

Xylocopa erratica Smith, 1874:293. Brèthes, 1916: 411 (sin.); Hurd, 1955: 62-63.

Distribuição. BRASIL. Goiás. Minas Gerais. Espirito Santo. Rio de Janeiro. São Paulo. Paraná. Santa Catarina. Rio Grande do Sul: Alegrete, Canela, Capão da Canoa, Guaíba, Nova Petrópolis, Osório, Piratini, Planalto, Porto Alegre, Rio Grande, São Francisco de Paula, São Lorenço do Sul, Tenente Portela, Viamão. PARAGUAI URUGUAI. ARGENTINA.

Flores visitadas. Alstroemeria isabellana (Alstroemeriaceae), Hippeastrum sp. (Amaryllidaceae), Aspilia montevidensis (Asteraceae), Justicia brasiliana (Acanthaceae), Opuntia monacantha (Cactaceae), Canna indica (Cannaceae), Crotalaria tweediana (Fabaceae), Malvaviscus arboreus (Malvaceae), Tibouchina gracilis (Melastomataceae), Fuchsia regia (Onagraceae), Passiflora caerulea (Passifloraceae), Solanum sisymbriifolium, S. vaillantii, S. variabile (Solanaceae).

Nidificação. Em entrenós de bambu.

Xylocopa (Xylocopoda) Hurd \& Moure, 1963 Xylocopa (Xylocopoda) Hurd \& Moure, 1963:105 (espécie-tipo: Xylocopa elegans Hurd \& Moure, 1963, por designação original).

Notas taxonômicas. Subgênero com duas espécies descritas, uma do Paraná e outra de Minas Gerais, esta por FrIESE (1925), com o nome de $X$. madida. São espécies raras, pouco documentadas.

17.- Xylocopa (Xylocopoda) elegans Hurd \& Moure, 1963 Xylocopa (Xylocopoda) elegans Hurd \& Moure, 1963:295. Sul.

Distribuição. BRASIL. Paraná. Rio Grande do Sul: Caçapava do

Flores visitadas. Solanum sisymbriifolium (Solanaceae).
Nidificação. Não conhecida.

Xylocopa (Xylocopsis) Hurd \& Moure, 1963

Xylococpa (Xylocopsis) Hurd \& Moure, 1963:124 (espécie-tipo: Xylocopa funesta Maidl, 1912, por designação original).

18.- Xylocopa (Xylocopsis) funesta Maidl, 1912 Xylocopa funesta Maidl, 1912:321.

Xylocopa (Xylocopsis) funesta; Hurd \& Moure, 1963:127.

Xylocopa plaumanni Moure, 1951:320; Hurd \& Moure, 1963: 313 (sin.).

Distribuição. BRASIL. Paraná. Santa Catarina. Rio Grande do Sul: Caçapava do Sul, Santana da Boa Vista, São Francisco de Paula. PARAGUAI. ARGENTINA. Misiones.

Flores visitadas. Pithecoctenium echinatum (Bignoniaceae), Tibouchina gracilis (Melastomataceae), Passiflora caerulea (Passifloraceae), Styrax leprosus (Styracaceae).

Nidificação. Em madeira morta, em parte úmida da Floresta.

Comportamento. Espécie rara de mamangabas grandes, ocorrendo na Serra de Sudeste e na Serra Geral e na Serra do Mar no Paraná. Pela manhã os machos voam com grande velocidade em territórios extensos de $1 \times 2 \mathrm{~m}$ até 1 x $10 \mathrm{~m}$ sobre vegetação baixa sem flores, formando faixas irregulares, mudando de velocidade e direção muitas vezes. Apresentam comportamento agressivo entre si e atacam todos os invasores de território, inclusive pássaros pequenos. Repetidamente pousam num galho esfregando o metasoma, provavelmente para marcação de território com feromônios.

Notas taxonômicas. Subgênero monotípico. Moure viu os tipos de X. funesta em 1958, em Viena, podendo sinonimizar X. plaumanni.

Xylocopa (Xylocospila) Hurd \& Moure, 1963

Xylocopa (Xylocospila) Hurd \& Moure, 1963:109 (espécie-tipo: Xylocopa bambusae Schrottky, 1902, por designação original).

19.- Xylocopa (Xylocospila) bambusae Schrottky, 1902 Xylocopa bambusae Schrottky, 1902:462;475.

Xylocopa (Xylocospila) bambusae; Hurd \& Moure, 1963:113. Xylocopa eburnea Friese, 1903:202; Hurd \& Moure, 1961: 186 (sin.). Xylocopa bellula Brèthes, 1916:413; Hurd \& Moure, 1961: 187 (sin.).

Distribuição. BRASIL. Santa Catarina. Rio Grande do Sul: Caçapava do Sul e Santana da Boa Vista. PARAGUAI. ARGENTINA. Misiones.

Flores visitadas. Justicia brasiliana (Acanthaceae), Baccharis articulata (Asteraceae), Buddleja thyrsoides (Buddlejaceae), Glechon thymoides (Lamiaceae).

Nidificação. Em entrenós de bambu ou taquara.

Notas taxonómicas. Subgênero monotípico. Os exemplarestipo foram descobertos no Rio Grande do Sul por von Ihering e em Misiones por Brèthes, 1916, que a descreveu como $X$. bellula. Espécie rara, registrada com somente poucos exemplares no estado. MincKLey (1998) e Michener (2000) 
incluíram o subgênero em Schonnherria, apesar da falta de colorido metálico nas fêmeas, inteiramente pretas, e os machos com cores únicas entre os Xylocopinae. Os machos são uma exceção entre todas as Xylocopa pelo colorido ebúrneo dos segmentos I-V do abdome.

\section{Chave para identificação das espécies de Xylocopa do Rio Grande do Sul}

A chave foi feita para as fêmeas e machos ocorrentes no Rio Grande do Sul e baseia-se, com pequenas modificações, na apresentada por HuRD \& MOURE (1963:95-98), retirados os subgêneros não ocorrentes e acrescentados os dados para identificação das espécies, quando mais de uma.

A chave abrange 19 espécies. As fêmeas de $X$. (Ioxylocopa) não são conhecidas.

\section{Fêmeas}

1. Área frontal larga, sem carena ou tubérculo médios, ladeada por carenas divergentes para cima; ocelos posteriores situados acima da tangente orbital superior, com carenas subjacentes, curtas ou alongadas em cornos; clípeo com os cantos ânterolaterais fortemente protuberantes; com ou sem faixas tergais avermelhadas. Grandes, sobrepassando os $30 \mathrm{~mm}$

3. X. (Megaxylocopa) frontalis

Área frontal com uma carena média forte terminando mais alta pouco acima dos alvéolos, ou ao menos um tubérculo médio; sem cornos ou carenas laterais nem subjacentes aos ocelos; clípeo com os cantos ântero-laterais chatos no plano facial. Não sobrepassando os $30 \mathrm{~mm}$

2(1). Dente apical externo da mandíbula mais estreito que o interno e com o ápice alongado-arredondado, o interno mais largo e com o ápice truncado em linha reta; tamanho $16-17 \mathrm{~mm}$.....

16. X. (Stenoxylocopa) artifex

Dente apical externo da mandíbula tão ou mais largo que o interno, este com o ápice arredondado; tamanho variável 3

3(2). Esternos abdominais com uma carena elevada ao longo do meio, terminando em forte ângulo no bordo de cada esterno; sutura látero-epistomal em forma de sino, separando o clípeo das áreas paroculares; margem apical de E1 inteira ...X.(Neoxylocopa). .4

Esternos abdominais planos ou com fraca carena sem formar ângulo no bordo de cada esterno; sutura epistomal lisa sem forma de sino, separando o clípeo das áreas paroculares; margem apical de E1 chanfrada 10

4(3). Com revestimento piloso vermelho-ferrugíneo no dorso do tórax, ou em franja aos lados do abdome; de resto preto-pilosas 5

Toda a pilosidade uniformemente preta, no máximo uma pequena mancha sanguínea logo abaixo do término da placa basitibial

5(4). Dorso do tórax vermelho-ferrugíneo piloso, o resto do corpo preto-piloso; placa labral tridentada 11.X. (Neoxylocopa) tacanensis

Abdome com franjas laterais vermelho-ferrugíneas, o resto do corpo preto-piloso; placa labral apenas com pequeno dente médio, os bordos laterais simples 5.X. (Neoxylocopa) augusti

6(4). Tergos com faixas tegumentares avermelhadas, às vezes fracas; asas pretas com forte brilho violáceo . 8. X. (Neoxylocopa) nigrocincta

Tergos uniformemente pretos; asas pretas com forte brilho violáceo, ou claro-pardacentas com reflexos cúpreos 7

7(6). Asas pardo-claras semi-transparentes, com reflexos cúpreos a bronzeados 8

Asas escuras com reflexos azul-violáceos ou esverdeados 9

8(7). Tíbias posteriores com uma pequena mancha sanguínea logo abaixo do ápice da placa basitibial formada por cerdas vermelhas; no restante preto-pilosas 7.X. (Neoxylocopa) haematospila

Tíbias posteriores inteiramente preto-pilosas, sem essa mancha sanguínea abaixo da placa basitibial

6. X. (Neoxylocopa) brasilianorum

9(7). Asas pardo-escuras com forte reflexo esverdeado; T2 com a pontuação muito esparsa no disco, os intervalos entre os pontos maiores que $4 \mathrm{dp}$........ 10. X. (Neoxylocopa) suspecta Asas pardo-escuras com forte reflexo violáceo; T2 com a pontuação mais densa, no disco os pontos cerca de $2 \mathrm{dp}$, ou pouco mais

9.X. (Neoxylocopa) ordinaria

10(3). Órbitas subparalelas ou convergentes em baixo 11

Órbitas convergentes em cima 13

11 (10). Fronte inteiramente plana com um pequeno tubérculo pouco abaixo do meio, sem ligação careniforme ao ocelo médio; clípeo mais longo que a distância clipeo-ocelar; grádulo de T1 continuado aos lados do tergo .......... 4. X. (Nanoxylocopa) ciliata

Fronte com uma carena desde o ocelo médio elevandose até pouco acima da tangente alvéolo-antenal; clípeo mais curto que a metade da sua largura; grádulo basal de T1 terminando aos lados sem acompanhar os bordos do tergo 
12(11). Placa basitibial com o ápice bífido assimétrico, a projeção anterior dentiforme, a posterior lobada; genas lisas e brilhantes com pontuação esparsa; esparsa também aos lados dos sulcos parapsidais 19. X. (Xylocospila) bambusae

Placa basitibial com o ápice bífido quase simétrico, as duas projeções dentiformes; genas mates com pontuação esparsa; pontuação densa aos lados dos sulcos parapsidais

17.X. (Xylocopoda) elegans

13(10). Flagelo muito mais longo que o comprimento máximo do olho; T3-4 com grádulo; tegumento preto, quase sem brilho metálico ... 14

Flagelo no máximo tão longo como o comprimento do olho; T2-6 sem grádulo; tegumento fortemente verde dourado até azul ou violáceo metálico ...... X. (Schonnherria). . 15

14(13). Clípeo curto, menor que a metade da sua largura; pilosidade abdominal toda preta, moderadamente esparsa, os pêlos no disco dos tergos chegando ao ponto seguinte ........ 18.X. (Xylocopsis) funesta Clipeo tão longo como a metade da sua largura; pilosidade dos tergos longa e densa, os pêlos sobrepassando a origem do seguinte; nos lados dos dois últimos tergos e esternos com um feixe destacado de pêlos brancos a amarelentos 1.X. (Dasyxylocopa) bimaculata

15(13). Abelhas maiores; asa mais longa que $15 \mathrm{~mm}$ 16 Abelhas menores; asa mais curta que $13 \mathrm{~mm}$ 17

16(15). E4-5 com cerdas brancas aos lados; tergos sem faixa tegumentar avermelhada

12.X. (Schonnherria) macrops

E4-5 sem cerdas brancas laterais; ao menos alguns tergos com faixa tegumentar avermelhada ........ 13.X. (Schonnherria) simillima

17(15). Tegumento de um azul-violáceo intenso 14. X. (Schonnherria) splendidula Tegumento esverdeado

15.X. (Schonnherria) varians

\section{Machos}

Machos de algumas espécies são ainda desconhecidos e, igualmente, a sua ligação às fêmeas.

1. Clípeo preto, se maculado com coloração pálida e vértice em vista frontal geralmente não elevado, no máximo levemente elevado acima do nível superior dos olhos; o ápice do escapo não ou só levemente ultrapassando o nível superior dos olhos (neste caso T1-T4 com manchas brancoamareladas); olhos alcançando a margem superior da cabeça em vista frontal ou a distância órbitooccipital muito menor que primeiro flagelômero; olhos freqüentemente grandes e muitas vezes fortemente convergentes em cima ......................... 2

Clípeo sempre com coloração pálida e o vértice em vista frontal fortemente elevado acima do nível superior dos olhos; ápice do escapo ultrapassando em muito o nível superior dos olhos; distância órbitooccipital longa, maior que primeiro flagelômero; olhos sempre pequenos, nunca fortemente convergentes em cima .8

2(1). Tégulas inteiramente pontuadas ou fortemente pubescentes ao longo da margem 3

Tégulas sem pontos, glabras e brilhantes, pelo menos no terço póstero-lateral

4

3 (2). T1-T4 com largas faixas branco-amareladas, às vezes reduzidas no T5; distância interalveolar mais curta que distância interocelar; suturas subantenais longas, mais longas que a distância interalveolar; escapo ultrapassando o nível superior dos olhos; margens póstero-laterais das tégulas densamente pilosas; margem apical de E1 inteira

19. X. (Xylocospila) bambusae

T1-T4 pretos; distância interalveolar mais longa que distância interocelar; suturas subantenais curtas, menores que a distância interalveolar; escapo não ultrapassando o nível superior dos olhos; margens póstero-laterais das tégulas sem pêlos; margem do E1 fortemente emarginada no meio

4.X. (Nanoxylocopa) ciliata

4(2). Escutelo de perfil achatado, não abaulado, no mesmo plano do metanoto e das margens ântero-dorsais do propódeo; propódeo exposto horizontalmente 17. X. (Xylocopoda) elegans

Escutelo de perfil abaulado, acima do nível do metanoto e propódeo; parte mediana do metanoto fortemente inclinada; propódeo em declive, não exposto horizontalmente ao longo da margem ântero-dorsal

.. 5

5(4). T3-T5 com grádulos, cada grádulo profundamente recortado, interrompido no meio e terminando no limite posterior do tergo; tegumento fracamente metálico ....................... 18.X. (Xylocopsis) funesta Tergos sem grádulos; tegumento com brilho metálico facilmente perceptível .........X. (Schonnherria). 6

6(5). Abelhas relativamente grandes, comprimento das asas maior que $15 \mathrm{~mm}$; olhos fortemente convergentes em cima, distância superior entre os órbitas aproximadamente do diâmetro do ocelo; esternos com desenhos amarelo-esbranquiçados

12. X. (Schonnherria) macrops 
Abelhas menores, comprimento das asas menor que $13 \mathrm{~mm}$; distância superior entre os olhos muito maior que o diâmetro do ocelo; esternos sem desenhos amarelo-esbranquicados . .7

7(6). Tegumento azul-violáceo intenso 14. X. (Schonnherria) splendidula Tegumento esverdeado ..15. X. (Schonnherria) varians

8(1). Mandíbula, em vista lateral, alargada no ápice, o dente externo apical projetado ou alargado para baixo da margem inferior da mandíbula .... ... 9

Mandíbula, em vista lateral, não alargada no ápice, o dente externo apical não projetado ou alargado para baixo

9(8). Tegumento ferrugíneo ou amarelado, pilosidade ferrugínea ou amarela abundante; tégulas ferrugíneas; escapo inteiramente ferrugíneo ou com uma estria longitudinal frontal amarela ..... 10

Tegumento predominantemente preto, pelo menos na superfície dorsal, coberto por pêlos escuros, esbranquipados ou cinzentos; tégulas pretas; escapo predominantemente ou inteiramente preto, sem estria longitudinal amarela

16. X. (Stenoxylocopa) artifex

10(9). Face muito larga, distância interorbital maior que o comprimento do olho; carena frontal substituída por um sulco longitudinal fraco; olhos mais convergentes embaixo

3.X. (Megaxylocopa) frontalis

Face estreita, distância interorbital menor que o comprimento do olho; carena frontal presente, contudo, às vezes muito fraca, seu ápice claramente elevado sobre o tegumento supraclipeal adjacente; olhos mais convergentes em cima.(Os machos do subgênero Neoxylocopa são muito semelhantes entre si e podem ser diferenciados somente através da genitália) .....

X. (Neoxylocopa)

11(8). Propódeo fortemente inclinado, quase sem superfície horizontal; basitarso posterior mais longo que as tíbias adjacentes

1.X. (Dasyxylocopa) bimaculata

Propódeo largamente abaulado e fortemente exposto ântero-dorsalmente; basitarsos posteriores mais curtos que as tíbias adjacentes

2.X. (Ioxylocopa) chrysopoda

Agradecimentos. Ao Dr. Albino M. Sakakibara (UFPR) e Dra. Luciana Ianuzzi (UFPE) pelas sugestões e revisão do manuscrito. Ao CNPq pelo apoio financeiro.

\section{REFERÊNCIAS}

Alves-Dos-SAntos, I. 1999. Abelhas e plantas melíferas da mata atlântica, restinga e dunas do litoral norte do estado do Rio Grande do Sul, Brasil. Revista Brasileira de Entomologia 43(3/4): 191-223.

Ashmead, W. H. 1899. Classifcation of the bees of the superfamiliy Apoidea. Transactions of the American Entomological Society 26: $49-100$.

BRÉTHes, J. 1916. Le genre "Xylocopoa" Latreille dans la République Argentine. Physis 2: 407-421.

Buchmann, S. L. 1983. Buzz pollination in angiosperms. p. 73-117. In: C. E. Jones \& R. J. Little (Ed.). Handbook of experimental pollination biology. New York, Van Nostrand Reinhold, 558 p.

Burmeister, H. 1876. Hymenopterologische Mittheilungen 1: Die Xylocopa-Arten des La Plata-Gebietes. Stettiner Entomologische Zeitung 37: 151-159.

Cockerell, T. D. A. 1911. Descriptions and records of bees - XXXV. Annals and Magazine of Natural History 7: 310-319.

Cockerell, T. D. A. 1926. Descriptions and records of bees - CXI. Annals and Magazine of Natural History 17: 657-665.

Cockerell, T. D. A. 1949. Bees from Central America, principally Honduras. Proceedings of the United States Natural Museum 98: 429-490.

Dalla Torre, C. G. 1896. Catalogus Hymenopterorum, Vol. 10. Apidae (Anthophila). Leipzig, Engelmann, 643 p.

Dusmet y Alonso, J. M. 1924. Las "Xylocopoa" (Hymen.: Apidae) en las colecciones de Madrid. Trab. Mus. Nac. Cienc. Nat. (Madrid), ser. Zoól. 49: 5-58.

Enderlein, G. 1913. Zur Kenntnis der Xylocopen Südamerikas und über einen Zwitter von Xylocopa ordinaria. Arch. Naturgeschichte (A) 79: 156-170.

Fabricius, J. C. 1804. Systema Piezatorum, xxx $+432 \mathrm{p}$.

FAegri, K. \& L. VAN DER PIJL. 1979. The principles of pollination ecology. Oxford, Pergamon, 244 p., $3^{\text {rd }}$ ed.

Friese, H. 1903. Neue Arten der Bienengattung Xylocopa Latr. aus der Neotropischen und Orientalischen Region (Hym.). Zeitschrift für Hymenopterologie und Dipterologie 3: 202-208.

Friese, H. 1925. Neue neotropische Bienenarten, zugleich II: Nachtrag zur Bienenfauna von Costa Rica. Stettiner Entomologische Zeitung 86 (2): 1-41.

Garcia, M. T. A. \& P. S. Hoc. 1997. Floral biology and reproductive system of Passiflora caerulea (Passifloraceae). Beitrage zur Biologie der Pflanzen 71: 1-21.

Gerling, D.; P. D. Hurd \& A. Hefetz. 1983. Comparative behavioral biology of two Middle East species of carpenter bees (Xylocopa Latreille) (Hymenoptera: Apoidea). Smithsonian Contributions to Zoology 369: 1-33.

Gerling, D.; H. H. W. Velthuis \& A. Hefetz. 1989. Bionomics of the large carpenter bees of the genus Xylocopa. Annual Review of Entomology 34: 163-190.

GRAF, V. 1965. Contribuição ao estudo da anatomia da cabeça dos Apoidea. - A musculatura do complexo lábio-maxilar de Xylocopa Latreille, 1802 (Hymenoptera-Apoidea). Boletim da Universidade Federal do Paraná, Zoologia 2(7): 93-100.

GRAF, V. 1968. Observações sôbre o canal salivar cefálico de alguns Apidae. Boletim da Universidade Federal do Paraná, Zoologia 3 (3): 65-78.

GRAF, V. 1972. Contribuição ao estudo da anatomia da cabeça dos Apoidea II - A musculatura do complexo lábio - maxilar. Boletim da Universidade Federal do Paraná, Zoologia 5: 139-173.

Harter, B. 1999. Bienen und ihre Trachtpflanzen im AraukarienHochland von Rio Grande do Sul, mit Fallstudien zur Bestäubung von Pionierpflanzen. Dissertação. Universidade de Tübingen, Selbstverlag, $185 \mathrm{p}$.

Hoffmann, M. 1994. Observações sobre a polinização entomófila de Helianthus annuus L. em Viamão, Rio Grande do Sul. Anais da Sociedade Entomológica do Brasil 23(3): 391-397.

Hoffmann, M. 1995. Abelhas nativas (Hymenoptera, Apoidea) numa área agrícola no Sul do Brasil e sua importância para a polinização de 
Phaseolus vulgaris (Leguminosae). Iheringia, Série Zoologia: 129-133.

Hogendoorn, K. 1994. Socio-economics of the carpenter bee Xylocopa pubescens. Dissertação, Universidade de Utrecht.

Hogendoorn, K. \& H. H. W. Velthuis, 1993. The sociality of Xylocopa pubescens: Does a helper really help? Behavioral Ecology Sociobiology. 32(4): 247-257.

Hogendoorn, K. \& H. H. W. Velthuis, 1995. The role of young guards in Xylocopa pubescens. Insect Sociaux 42(4): 427-448.

Hurd, P. D. 1955. The carpenter bees of California. Bulletin of the California Insect Survey 4: 35-72.

Hurd, P. D. 1978a. An annotated catalog of the carpenter bees (genus Xylocopa Latr.) of the Western Hemisphere (Hymenoptera, Anthophoridae). Smithsonion Institution Press, Washington D. C., $116 \mathrm{p}$.

Hurd, P. D. 1978b. Bamboo-nesting carpenter bees (genus Xylocopa Latreille) of the subgenus Stenoxylocopa Hurd and Moure. Journal of the Kansas Entomological Society 51: 746-764.

Hurd, P. D. JR. \& J. S. Moure. 1960. A new-world subgenus of bamboonesting carpenter bees belonging to the genus Xylocopa Latreiile. Annals of the Entomological Society of America 63: 809-821.

Hurd, P. D. JR. \& J. S. Moure. 1961. Systematics of the carpenter bee types (genus Xylocopa Latreille) contained in the collections of the Museo Argentino de Ciencias Naturales "Bernardino Rivadavia" (Hymenoptera: Apoidea). Journal of the Kansas Entomological Society 34(4): 181-195.

HuRD, P. D. JR. \& J. S. Moure. 1963. A classification of the large carpenter bees (Xylocopini) (Hymenoptera: Apoidea). University of California Publications in Entomology 29: 1-365.

Lepeletier de Saint-fargeau, A. L. M. 1841. Histoire naturelle des insectes. Hyménoptères Suites à Buffon. Paris, Roret 2: 1680 .

Linnaeus, C. 1767. Systema Naturae. Editio Duodecima Reformata. Holmiae, Laur. Salvii 12: 901-1068.

Linnaeus, C. 1775. In: Vollständiges Natursystem des C. v. Linné mit einer Erklärung. Nürnberg 5: 1-978.

Linsley, E. G., C. M. Rick \& S. G. STephens 1966. Observations on the floral relationships of the Galapagos carpenter bee (Hymenoptera, Apoidea). Pan-Pacific Entomologist 42: 1-18.

MaidL, F. 1912. Die Xylocopen (Holzbienen) des Wiener Hofmuseums: Ein Beitrag zu einer Monographie dieser Gattung. Annalen des Königlich-Kaiserlichen Naturhistorischen Hofmuseums (Wien) 26: 249-330.

Meunier, F. 1892. Observations sur quelques apides d'Ecuador. Jorn. Acad. Sci. Math. Phys. e Nat. (Lisboa) 2: 63-65.

Michener, C. D. 1954. Bees of Panamá. Bulletin of the American Museum of Natural History 104: 1-176.

Michener, C. D. 1974. The social behavior of the bees. A comparative study. Cambridge, Harvard University Press, $414 \mathrm{p}$.

Michener, C. D. 1997. Genus-group names of bees and supplemental family group names. Scientific Papers, Natural History Museum, University of Kansas 1: 1-81.

Michener, C. D. 2000. The bees of the world. Baltimore, London, The Johns Hopkins University Press, 913 p.

MinCKLEY, R. L. 1998. A cladistic analysis and classification of the subgenera and genera of the large carpenter bees, tribe Xylocopini (Hymenoptera: Apidae). Scientific Papers. Natural History Museum. The University of Kansas 9: 1-47.

Moure, J. S. 1949. Notas sôbre algunas abejas de Tacanas, Tucumán. Argentina, II (Hymenopt. Apoidea). Revista de Entomologia 20: 437-460.

MouRE, J. S. 1951. Tres espécies novas de Xylocopa Latreille (Hymenopt.Apoidea). Dusenia 2: 317-322.

Moure, J. S., 1960. Notes on types of neotropical bees described by Fabricius (Hymenoptera: Apoidea). Studia Entomologica 3: 97160.

Moure, J. S. \& J. M. F. Camargo. 1988. Uma nova espécie de Xylocopa (Neoxylocopa) do Brasil (Hymenoptera, Apoidea). Revista Brasileira de Entomologia 32(2): 219-214.

Olivier, G. A. 1789. Abeille, In: Encyclopédie Méthodique, histoire naturelle, Publiée par une société de gens de lettres, de savans et d'artistes. Insectes. Paris, Liége, 4: $331 \mathrm{p}$.

Pérez, J. 1901. Contribution à l'étude des Xylocopes. Actes de la Société Linnéenne de Bordeaux 56 (ser.6.vol.6): 1-128.

Roubik, D. W. 1995. Applied pollination in tropical America. Pollination of cultivated plants in the tropics. FAO Agricultural Services Bulletin 118, $198 \mathrm{p}$.

Sandhouse, G. A. 1943. The type species of the genera and subgenera of bees. Proceedings of the United States Natural Museum 92: 519-619

SAZima, I. \& M. SAzima. 1989. Mamangavas e irapuás (Hymenoptera, Apoidea): Visitas, interações e consequências para polinização do maracujá (Passifloraceae). Revista Brasileira de Entomologia 33: $119-134$.

Schlindwein, C. 1995. Wildbienen und ihre Trachtpflanzen in einer südbrasilianischen Buschlandschaft: Fallstudie Guaritas, Bestäubung bei Kakteen und Loasaceen. Ulrich Grauer, Stuttgart, $141 \mathrm{p}$

Schlindwein, C. 1998. Frequent oligolecty characterizings a diverse beeplant community in a xerophytic bushland of subtropical Brazil. Studies on Neotropical Fauna \& Environment 33: 46-59.

Schlumpberger, B. O. 1996. Fortpflanzungsbiologie von Holzbienen im Araukarienwald; Funktion von Pheromondrüsen bei Männchen von Xylocopa bimaculata. Diplomarbeit Universidade de Tübingen, $78 \mathrm{p}$.

Schlumpberger, B. O. \& D. Wittmann. 2000. New odour glands in Xylocopa males (Hymenoptera: Apoidea: Anthophoridae). Journal of Hymenopterological Research 9(2): 363-369.

Scholz, E. 1988. Artenspektrum, Verbreitung, Bionomie und Paarungsbiologie von Holzbienen (Apoidea: Xylocopinae) in Rio Grande do Sul, Südbrasilien. Diplomarbeit, Universidade de Tübingen, $58 \mathrm{p}$.

Schremmer, F. 1972. Der Stechsaugrüssel, der Nektarraub, das Pollensammeln und der Blütenbesuch der Holzbienen (Xylocopa) (Hymenoptera, Apidae). Zeitschrift fuer Morphologie der Tiere 72: 263-294.

Sснготтку, C. 1901. Biologische Notizen solitärer Bienen von S. Paulo (Brasilien). Allgemeine Zeitschrift für Entomologie 6: 209216.

Schrotткy, C. 1902. Ensaio sobre as abelhas solitárias do Brasil. Revista do Museu Paulista 5: 330-613.

Sмiтн, F. 1854. Catalogue of the hymenopterous insects in the collection of the British Museum, pt. 2, Apidae. London, 199465 .

Sмiтh, F. 1874. Monograph of the genus Xylocopa Latr. Transactions of the Entomological Society of London: $247-302$

Taschenberg, E. 1879. Die Arten der Gattung Xylocopa Ltr. des Halle'schen Zoologischen Museums. Zeitschrift f. d. Gesamt. Naturwissenschaften 52: 563-599.

Urban, D. 1963. Estudo comparativo da origem de alguns músculos cefálicos das abelhas (Hymenoptera-Apoidea). Boletim da Universidade Federal do Paraná, Zoologia 2(7): 21-33.

VAn Der PiJl, L. 1954. Xylocopa and flowers in the tropics. Proceedings of the Koninklijke Nederlandse Akademie von Wetenschappen Natural Sciences 57: 413-423, 541-562.

Velthuis, H. H. W. \& D. GerLing. 1983. At brink to sociality: interactions between adults of the carpenter bee Xylocopa pubescens Spinola. Behavioral Ecology and Sociobiology 12: 209-214.

WATMOUGH, R. H. 1974. Biology and behavior of carpenter bees in Southern Africa. Journal of the Entomological Society of Southern Africa 37: 261-281.

Watmough, R. H. 1983. Mortality, sex ratio and fecundity in natural populations of large carpenter bees (Xylocopa ssp.). Journal of Animal Ecology 52: 111-125.

Wittmann, D. \& M. Hoffmann. 1990. Bees of Rio Grande do Sul, southern Brazil (Insecta, Hymenoptera, Apoidea). Iheringia, Série Zoologia 71: 17-43.

Wittmann, D. \& E. Scholz. 1989. Nectar dehydration by male carpenter bees as preparation for mating flights. Behavioral Ecology and Sociobiology 25: 387-391. 